\title{
Diagnosis and Treatment of Carpal Tunnel Syndrome in A Tertiary Care Center in Mexico City
}

\author{
I Campos-Serna, Jaime Aron Garcia-Espinoza*, Alberto Ignacio Cahuana-Quispe, \\ Cuahutemoc Marquez-Espriella, Marco Antonio Cuervo-Vergara, Jose Carlos Martinez- \\ Lopez, Rodrigo Dávila-Diaz
}

Division of Plastic and Reconstructive Surgery, Hospital Central Sur de Alta Especialidad de Petróleos Mexicanos, Mexico City, Mexico

*Corresponding Author:

Jaime Aron Garcia Espinoza, MD; Division of Plastic and Reconstructive Surgery, Hospital Central Sur de Alta Especialidad de Petróleos Mexicanos, Anillo Periférico 4091,

Colonia Fuentes del Pedregal, Delegación Tlalpan, ZC 14140, Mexico City, Mexico.

Tel: $+52-5530588839$

Email: Jaime_506@yahoo.com.mx

Received: August 17, 2019

Revised: March 1, 2020

Accepted: March 10, 2020

\section{ABSTRACT}

\section{BACKGROUND}

Carpal tunnel syndrome is the most common peripheral neuropathy affecting patients at productive age and has an important economical impact on those who suffer it. This study assessed the diagnostic performance of carpal tunnel syndrome's signs and described the epidemiology at a tertiary care center in Mexico City.

\section{METHODS}

All patients diagnosed with carpal tunnel syndrome during a fiveyear period were included. Demographic data, electromyography results, positive clinical signs and the severity score according to the Italian scale were recorded. Diagnostic accuracy of Tinel and Phalen's signs were calculated via odds ratio.

\section{RESULTS}

Totally, 650 patients were diagnosed and treated during a fiveyear period, $84 \%$ were female and $16 \%$ male, and the mean age was 55.8 years. The associated comorbidities were trigger finger (36.1\%), thyroid disease (25.6\%) and diabetes (20\%). Diagnosis yielded for Phalen and Tinel signs were variable in each of the study groups (males and females) and showed to be beneficial in diagnosis of the disease.

\section{CONCLUSION}

Carpal tunnel syndrome is a complex disease in which clinical signs remain the cornerstone of diagnosis. Extension studies are useful to assess the severity of the disease.

\section{KEYWORDS}

Carpal tunnel syndrome; Phalen; Tinel; Neuropathy

Please cite this paper as:

Campos-Serna I, Garcia-Espinoza JA, Cahuana-Quispe AI, MarquezEspriella C, Cuervo-Vergara MA, Martinez-Lopez JC, Dávila-Diaz R. Diagnosis and Treatment of Carpal Tunnel Syndrome in A Tertiary Care Center in Mexico City. World J Plast Surg 2020;9(2):213-218. doi: 10.29252/wips.9.2.219.

\section{INTRODUCTION}

Carpal tunnel syndrome is the most frequent peripheral mononeuropathy worldwide with an estimated prevalence of 1 to $5 \%$ and an incidence of 2.2 to $5.4 \%$ per 1000 people. ${ }^{1}$ Although its pathogenesis is multifactorial, a higher prevalence and incidence has been found in women because they have a narrower carpal 
tunnel prone to higher internal pressures that cause neural compression. ${ }^{2}$ Identified risk factors for this disease are obesity, female gender ${ }^{3}$ and comorbidities such as diabetes mellitus [Odds Ratio (OR): 2.2], ${ }^{4}$ hypothyroidism (OR: 1.4), ${ }^{5}$ rheumatoid arthritis (OR: 2.2$)^{6}$ and the use of drugs such as Anastrazole (OR: 2.6). ${ }^{7}$

Other factors associated are vicious positions of the wrist and repetitive activities with the upper extremities, although a statistical relation has not been proven. Neural damage in carpal tunnel syndrome is developed due to the increased pressure inside the tunnel. It disrupts axonal transport and compromises perineural irrigation causing ischemia of the median nerve. This phenomenon has physical and psychological implications. It mostly affects patients in their productive years of life, thus representing significant economical losses. ${ }^{8}$

Despite medical advances, the gold standard for diagnosis is a thorough clinical history and physical exam. The classic syndrome is described with intermittent paresthesias of the middle and ring finger, it mostly occurs at night, as well as dysesthesias that wakes the patient up., ${ }^{9} 10$ Severe manifestations such as loss of sensation and muscle atrophy of the thenar region, appear later in the course of the disease. In the physical examination, pain on palpation is often found. Tinel and Phalen maneuvers increase internal pressure in the tunnel evoking paresthesia in the territory of the median nerve, with a sensibility of $42-85 \%$ for Phalen sign and $38-100 \%$ for Tinel sign, and a specificity of $54-98 \%$ and $55-100 \%$, respectively. ${ }^{11}$

Diagnostic tools are not needed for the diagnosis, they only assist confirming it. Yet, electromyography (EMG) allows to rule out other causes of neural damage and establishes the degree of axonal injury in a systematic and concise way. ${ }^{12}$ Other tools such as wrist ultrasound have an acceptable diagnostic performance, but require specialized equipment and medical staff that results in higher costs..$^{13}$ Treatment continues to be an important point of study, conservative strategies such as postural changes or medical treatment with gabapentin have failed to show completely effective in the resolution of symptoms in the short and long term. ${ }^{14}$

Surgical release of the transverse ligament of the carpal tunnel is the treatment of choice for most cases, it has proven to stop axonal degeneration and has a therapeutic effect in the long term (up to two years) compared with the use of local steroids. Surgical treatment includes the conventional approach with an open incision in the volar aspect of the hand, and the endoscopic approach with a minimally invasive incision trough which a blade is inserted to release the ligament under direct vision. ${ }^{15}$

Studies show the latter to be less painful and to have a shorter recovery time. Yet, it requires highly expensive and specialized equipment, as well as experienced staff in order to be safe and effective. ${ }^{16}$ The research and teaching ethics committee of our institution approved the study with number 46/19HCSAE and did not apply the informed consent of patients, since it was a retrospective study where the identity of the patients was protected. This study was undertaken to describe the epidemiological profile of the population with diagnosis of carpal tunnel syndrome treated at a tertiary care center in Mexico City (age, gender, affected side, risk factors, comorbidities, EMG results). Also, the sensibility, specificity, positive predictive value (PPV) and negative predictive value (NPV), a probability of occurrence for Odds Ratio (OR) among clinical signs and electrical conduction studies, as well as the diagnostic accuracy of clinical signs (Phalen, Tinel and Durkan) were determined.

\section{MATERIALS AND METHODS}

The study includes patients with diagnosis of carpal tunnel syndrome treated in "Hospital Central Sur de Petroleos Mexicanos" in Mexico City, Mexicoa during 5-year period. Epidemiologic variables such as age, gender, affected side, bilaterality, sensibility, specificity, PPV and NPV, diagnostic accuracy and OR of clinical signs were compared with electric conduction studies. Severity of disease, history of local steroids, physical therapy, comorbidities, length of symptoms, number of procedures, recovery time, postoperative complications and need of reintervention were also described.

\section{RESULTS}

Totally, 650 patients treated during five years in our unit were included, $84 \%$ were female and $16 \%$ male, and the average age was 55.8 years, with a range of 48 to 63 years. The evolution of the disease had an average of 5.86 months 
with a range of 3 to 8 months. Our population presented concomitant comorbidities as trigger finger (36.1\%), thyroid diseases $(25.6 \%)$ and diabetes $(20 \%)$. The patients were divided in two groups according to gender, as female group having an average age of 55.1 years with a range of 45.2 to 62.7 years and an average evolution of 5.8 months with a range of 3 to 7 months. Likewise, $61 \%$ of these patients underwent surgical treatment and had a mean postoperative recovery of 12 days with a range of 11 to 14 days, and unilateral condition was found in $61 \%$ and bilateral in $39 \%$ of cases.

In the male group, the average age was 59.27 years old with a range of 52 to 65 years. They presented an evolution of 7.57 months with a range of 4.5 to 9 months, $52 \%$ of these patients underwent surgical treatment and had a 13-day mean postoperative recovery with a range of 8 to 14 days. Unilateral disease was present in $62 \%$ and bilateral in $38 \%$. All of the patients underwent electromyographic examination and was compared with the Italian clinical classification.

In the Female group it was found that $62 \%$ had the electrodiagnostic of neuropraxia and some cases were reported with neurotmesis (Figure 1). When compared with the Italian clinical classification, it was found that the vast majority presented clinical data compatible with incipient degrees of the disease (Figure 2). In the male group, it was observed that $61 \%$ had an electrodiagnostic of neuropraxia, 30\% axonotmesis and no cases of neurotmesis (Figure 3).

When measuring the severity of the disease with the Italian clinical classification, more than $50 \%$ presented grade IV and V of severity. However, when trying to correlate both ways of measuring the severity of the disease (clinical and electromyography), there was not any correlation between groups. When we compared diagnostic performance of the signs with electrodiagnosis; in the female group, we found a sensitivity of 72.8 , specificity of 43.7 , PPV of 89.2, NPV of 73.6, an OR of 2.9 and a diagnostic accuracy test of 0.6 (Figure 4).

For the Phalen sign, a sensitivity of 69 , specificity of 44.4, PPV of 89.9, negative predictive value of 84.4 , an OR of 2.8 and a diagnostic accuracy test of 0.67 . In the male group, we found a sensitivity of 66.6 , specificity of 50, PPV of 87.5, NPV of 77.7, OR of 2 and a diagnostic accuracy test of 0.56 . For the Phalen

\section{Female group Severity according to Electromyography}

neuroapraxia $\quad$ neurotnmesis $\quad$ axonotmesis normal

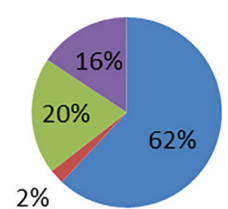

Fig. 1: Severity according to electromyographyc test.

\section{Female group Italian severity Scale}

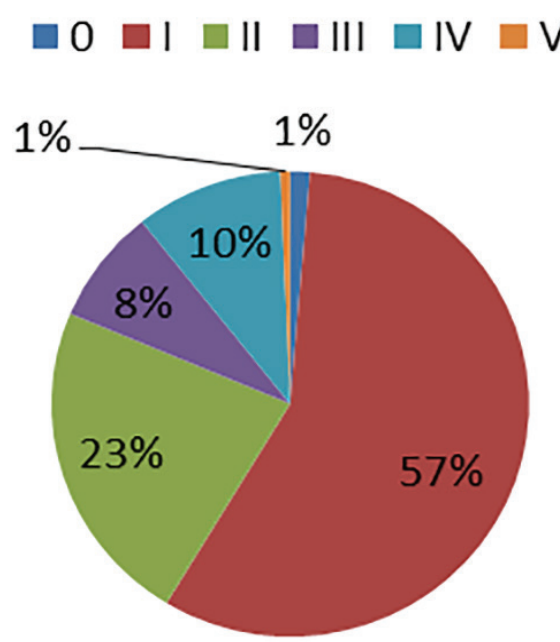

Fig. 2: Severity according to Italian scale in female group.

\section{Male group Severity according to Electromyography}

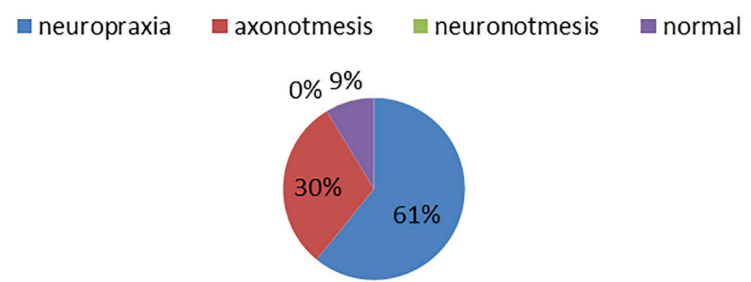

Fig. 3: Severity according to electromyographyc test. sign we found a sensitivity of 65 , specificity of 50 , positive predictive value 92.8 , NPV of 86.9 , an OR of 3.7 and a diagnostic accuracy test of 0.6.

\section{DISCUSSION}

Carpal tunnel syndrome is one of the most 


\section{Male group Italian severity scale}

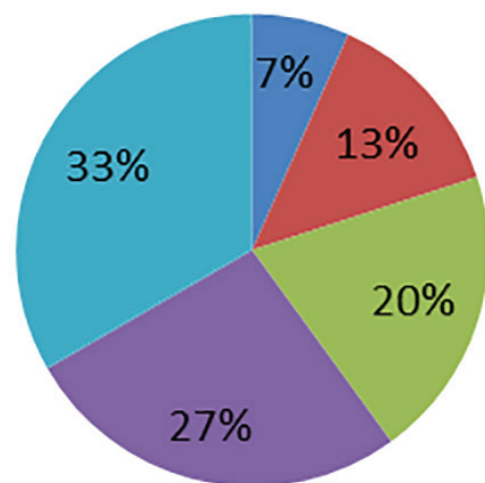

Fig. 4: Severity according to Italian scale in male group.

frequent complaints for a hand surgeon. Functional prognosis of the hand is based on the median nerve compression chronicity; that's why clinical data is crucial for an early detection and treatment. Our study showed similar results observed in other series regarding female gender predominance as a result of a narrower carpal tunnel. ${ }^{17}$ Mack et al. in their series reported an incidence of $74 \%{ }^{18}$ and Destefano et al. reported a $63 \%$ female predominance, respectively; when compared to our findings of $84 \%$. Regarding age range, they also obtained similar results demonstrating that this pathology affected the productive ages of life and impacted the economy of society. ${ }^{19}$

In ourstudy, the evolution of the condition from the onset of symptoms to surgery had an average of 5.86 months with a range of 3 to 8 months. Our population presented as comorbidities, thyroid diseases $(25.6 \%)$ and diabetes mellitus (20\%), in contrast to Destefano's series that showed only 2 and 5\%, respectively. In our population, there was a particular association of trigger finger in the same neuropathic hand in $36.1 \%$, which led us to think of a probable pathological association between different pathologies that afflicted the hand, so it is necessary to conduct studies that allow us to determine the strength of association between these pathologies and which of them could be the trigger. ${ }^{19}$

We decided to make two groups according to gender, because there are currently no other studies that allow us to identify differences regarding age in the natural history of this pathology. Our results are similar to other researchers, ${ }^{18}$ however, we found differences in the early onset of symptoms in women and a greater need for surgery in less evolution time (Table 1). Electrodiagnostic study is an adjuvant tool and not all patients need its performance; since a good medical history and physical examination is enough to make diagnosis and to start treatment. Electrodiagostic assessment is needed for staging and supporting a work disability (in active workers).

Neuropraxia was found in $62 \%$ and $61 \%$, respectively and it contrasted with the Italian clinical classification, in which women presented incipient stages and men severe stages; however, at the time of the clinicalelectrodiagnostic correlation, they showed no statistical significance between groups. In a systematic review, sensitivities ranging from $42 \%$ to $85 \%$ were illustrated for the Phalen sign and $38 \%$ to $100 \%$ for the Tinel sign and the specificity ranged from $54 \%$ to $98 \%$ and 55 to $100 \%$, respectively. ${ }^{11}$

The results obtained in this study demonstrated weak sensitivity and specificity measurements; however, the calculation of the predictive values in each of them demonstrated utility to confirm the diagnosis in a clinical ground, in the same way the OR calculations allowed us to establish the use of both clinical signs for diagnosis. ${ }^{11}$ What we observed when analyzing the data was a greater diagnostic performance of the Tinel sign for the women's group and a greater diagnostic performance of the Phalen sign for men (Table 2).

Carpal tunnel syndrome continues to be the most frequent compression neuropathy, with the female gender being the most affected. The highest incidence peak affects productive ages despite gender, so an early diagnosis is essential to reduce institutional costs that condition this pathology. The clinical signs of Tinel and Phalen remain fundamental elements of the diagnosis of the disease, despite their variable diagnostic performance, they allow to establish the existence of neural compression, when used together.

Electromyography is used to establish the neural damage type that exists. Despite reports of high diagnostic performance of the Durkan sign, in our group it was not explored, leaving a 
Table 1: Epidemiological and demographic variables

\begin{tabular}{llll}
\hline Variable & & $\begin{array}{l}\text { Female } \\
\mathbf{( 8 4 \% )}\end{array}$ & $\begin{array}{l}\text { Male } \\
\mathbf{( 1 6 \% )}\end{array}$ \\
\hline Age & Mean & 55.1 & 59.2 \\
& IQR & $45.2-62.7$ years & $52-65$ years \\
Time of evolution & Mean & 5.8 & 7.5 \\
& RIC & $3-7$ months & $4.5-9$ months \\
Surgical treatment & & $61 \%$ & $52 \%$ \\
Recovery time & Mean & 12 days & 13 days \\
& IQR & $11-14$ days & $8-14$ days \\
Laterality & Unilateral & $61 \%$ & $62 \%$ \\
& Bilateral & $39 \%$ & $38 \%$ \\
\hline
\end{tabular}

IQR: intercuartile range

Table 2: Diagnostic performance of clinical signs by group

\begin{tabular}{llll}
\hline Variable & & Female & Male \\
\hline Tinel sign & Sensibility & 72.8 & 66.6 \\
& Specificity & 43.7 & 50 \\
& Positive predictive value & 89.2 & 87.5 \\
& Negative predictive value & 73.6 & 77.7 \\
& Likelihood of occurrence (OR) & 2.9 & 2 \\
Phalen sign & Diagnostic accuracy test & 0.6 & 0.56 \\
& Sensibility & 69 & 65 \\
& Specificity & 44.4 & 50 \\
& Positive predictive value & 89.9 & 92.8 \\
& Negative predictive value & 84.4 & 86.9 \\
& Likelihood of occurrence (OR) & 2.8 & 3.7 \\
& Diagnostic accuracy test & 0.67 & 0.6 \\
\hline
\end{tabular}

line of research to determine its effectiveness in a Latin American population. For this reason, new lines of research should be created to establish associations with other hand pathologies, effective screening tests, medical care protocols in specialized centers and surgical techniques that help reducing the days of disability caused by the surgical resolution of this pathology.

\section{CONFLICT OF INTEREST}

The authors declare no conflict of interest.

\section{REFERENCES}

1 Pourmemari MH, Heliovaara M, ViikariJuntura E, Shiri R. Carpal tunnel release: Lifetime prevalence, annual incidence, and risk factors. Muscle Nerve 2018;58:497-502. doi: 10.1002/mus.26145.

2 Keir PJ, Rempel DM. Pathomechanics of peripheral nerve loading. Evidence in carpal tunnel syndrome. J Hand Ther 2005;18:25969. doi: 10.1197/j.jht.2005.02.001.
3 Bland JD. The relationship of obesity, age, and carpal tunnel syndrome: more complex than was thought? Muscle Nerve 2005;32:527-32. doi: 10.1002/mus.20408.

4 Albers JW, Brown MB, Sima AA, Greene DA. Frequency of median mononeuropathy in patients with mild diabetic neuropathy in the early diabetes intervention trial (EDIT). Tolrestat Study Group For Edit (Early Diabetes Intervention Trial). Muscle Nerve 1996;19:140-6. doi: 10.1002/ (SICI)1097-4598(199602)19:2<140::AIDMUS3>3.0.CO;2-E.

5 Shiri R. Hypothyroidism and carpal tunnel syndrome: a meta-analysis. Muscle Nerve 2014;50:879-83. doi: 10.1002/mus.24453.

6 Shiri R. Arthritis as a risk factor for carpal tunnel syndrome: a meta-analysis. Scand J Rheumatol 2016;45:339-46. doi: 10.3109/03009742.2015.1114141.

7 Sestak I, Sapunar F, Cuzick J. Aromatase inhibitor-induced carpal tunnel syndrome: results from the ATAC trial. $J$ Clin Oncol 2009;27:4961-5. doi: 10.1200/ 
JCO.2009.22.0236.

8 Shiri R, Heliovaara M, Ahola K, KailaKangas L, Haukka E, Kausto J, Saastamoinen P, Leino-Arjas P, Lallukka T. A screening tool for the risk of disability retirement due to musculoskeletal disorders. Scand J Work Environ Health 2018;44:37-46. doi: 10.5271/ sjweh.3684.

9 Bland JD. Carpal tunnel syndrome. BMJ 2007;335:343-6. doi: 10.1136/ bmj.39282.623553.AD.

10 Padua L, Padua R, Nazzaro M, Tonali P. Incidence of bilateral symptoms in carpal tunnel syndrome. J Hand Surg Br 1998;23:6036. doi: 10.1016/s0266-7681(98)80010-7.

11 MacDermid JC, Wessel J. Clinical diagnosis of carpal tunnel syndrome: a systematic review. J Hand Ther 2004;17:309-19. doi: 10.1197/j.jht.2004.02.015.

12 Jablecki CK, Andary MT, Floeter MK, Miller RG, Quartly CA, Vennix MJ, Wilson JR, American Association of Electrodiagnostic M, American Academy of N, American Academy of Physical M, Rehabilitation. Practice parameter: Electrodiagnostic studies in carpal tunnel syndrome. Report of the American Association of Electrodiagnostic Medicine, American Academy of Neurology, and the American Academy of Physical Medicine and Rehabilitation. Neurology 2002;58:1589-92. doi: 10.1212/wnl.58.11.1589.

13 Fowler JR, Gaughan JP, Ilyas AM. The sensitivity and specificity of ultrasound for the diagnosis of carpal tunnel syndrome: a meta-analysis. Clin Orthop Relat Res
2011;469:1089-94. doi: 10.1007/s11999-0101637-5.

14 Hui AC, Wong SM, Leung HW, Man BL, Yu E, Wong LK. Gabapentin for the treatment of carpal tunnel syndrome: a randomized controlled trial. Eur J Neurol 2011;18:726-30. doi: 10.1111/j.1468-1331.2010.03261.x.

15 Shi Q, MacDermid JC. Is surgical intervention more effective than non-surgical treatment for carpal tunnel syndrome? A systematic review. J Orthop Surg Res 2011;6:17. doi: 10.1186/1749-799X-6-17.

16 Atroshi I, Hofer M, Larsson GU, Ranstam J. Extended Follow-up of a Randomized Clinical Trial of Open vs Endoscopic Release Surgery for Carpal Tunnel Syndrome. JAMA 2015;314:1399-401. doi: 10.1001/ jama.2015.12208.

17 Márquez Espriella C, Sánchez-Medal FP, Heras JCL, Salomón CC, Palacios GG. Síndrome de túnel del carpo:¿ es el tiempo de evolución determinante en el resultado postquirúrgico? Cirugía Plástica 2012;22:1348.

18 Mack EM, Callinan NJ, Reams M, Bohn DC, Chmielewski TL. Patient-reported outcomes after open carpal tunnel release using a standard protocol with 1 hand therapy visit. $J$ Hand Ther 2017;30:58-64. doi: 10.1016/j. jht.2016.03.007.

19 DeStefano F, Nordstrom DL, Vierkant RA. Long-term symptom outcomes of carpal tunnel syndrome and its treatment. $J$ Hand Surg Am 1997;22:200-10. doi: 10.1016/S03635023(97)80152-9. 